\title{
Clinical Application of Optical Coherence Tomography in Patients with Non-ST-Elevation Acute Coronary Syndrome Combined with Intermediate Lesions
}

\author{
Hua Yu, MM, Likun Ma, MD, Kefu Feng, MM, Hongwu Chen, Hao Hu MM \\ Department of Cardiology, Anhui Provincial Hospital of Anhui Medical University, Hefei, China
}

\section{ABSTRACT}

Objective: This study aimed to evaluate the clinical significance and safety of optical coherence tomography (OCT) in patients with non-ST-elevation acute coronary syndrome (NSTEACS) combined with intermediate lesions.

Methods: Sixty-five NSTEACS patients with intermediate lesions confirmed with coronary angiography at our department were included in this study. Among them, 33 patients received only standardized drug treatment (drug group) and the other 32 patients received percutaneous coronary intervention (PCI) according to the OCT examination based on drug treatment (OCT group). Major adverse cardiovascular events (MACEs), revascularization, success rate of OCT examination, related complications, and other patient situations in the two groups during hospitalization and the 12-month follow-up period were compared.

Results: No death or stroke occurred in either group during hospitalization and follow-up. In the drug treatment group, six patients experienced frequent angina, and five patients with acute myocardial infarction were rehospitalized and underwent PCI procedures. In the OCT group, although two patients underwent repeat revascularization, no additional acute myocardial infarction events occurred. There was a statistically significant difference between the two groups (P $<.01)$. All patients in the OCT group successfully completed the related vessel examination, and 24 patients underwent PCI procedures because of unstable plaque diagnosed with OCT.

Conclusion: OCT-guided PCI is safe and effective for the treatment of patients with NSTEACS combined with intermediate lesions.

\section{INTRODUCTION}

Acute coronary syndrome (ACS) includes unstable angina (UA), non-ST-elevation myocardial infarction (NSTEMI), ST-elevation myocardial infarction (STEMI), and sudden death. In recent years, evidence-based medicine has shown that early interventional therapy for the middle- and highrisk groups of non-ST-elevation ACS (UA/NSTEMI/ NSTEACS) patients can significantly reduce the occurrence

Received November 20, 2015; received in revised form May 28, 2016; accepted fune 15, 2016.

Correspondence: Hua Yu, Department of Cardiology, Anhui Provincial Hospital of Anhui Medical University, 17 Lujiang Road, Luyang District, Hefei 230001, China; +86 13955169201; fax: +86055162282121 (e-mail: yblkcn@163.com). of death, myocardial infarction, stroke, and other major adverse cardiovascular events (MACEs) [Goldman 2012]. However, the use of percutaneous coronary intervention (PCI) for patients with vessel diameter stenosis at the critical level (50\%-70\%) of intermediate lesions [Fernandes 2007], as indicated by using coronary angiography (CAG), is controversial. Numerous studies have shown that the degree of coronary artery stenosis has no significant correlation with clinical outcomes. About $70 \%$ of coronary artery stenoses in patients with ACS were at a critical level [Rzeczuch 2005], whereas studies showed that $6 \%$ of patients with intermediate lesions in 1 year needed PCI because of acute coronary events, and $87 \%$ of these intermediate lesions requiring PCI had angiographic lesions accounting for $<60 \%$ of the intermediate lesions [Glaser 2005]. Intravascular ultrasound (IVUS) has been used to display cross-sectional images in real time and to assess the features of coronary plaques [Abizaid 1998; Takagi 1999; Briguori 2001] as well as to determine the fractional flow reserve. Assessment of the physiological significance of critical diseases from a functional point of view has become an important reference for the development of treatment strategies [Bech 2001; Tonino 2009; De Bruyne 2012]. Optical coherence tomography (OCT) is a recent medical imaging technique with a higher resolution than radiography, computed tomography (CT), magnetic resonance imaging (MRI), and ultrasound diagnostic techniques [Honda 2008]. This study was aimed at evaluating the safety and clinical significance of OCT in the management of NSTEACS patients with intermediate lesions in the middle-risk (thrombosis in myocardial infarction [TIMI] risk score 3-4) and high-risk (TIMI risk score 5-7) groups.

\section{MATERIALS AND METHODS}

\section{Subjects}

Sixty-five patients of the middle- and high-risk UA/ NSTEMI groups, hospitalized at our department between January 2012 and December 2012, who were found to have intermediate lesions located in a single vessel (except for the left main coronary artery [LMCA]) by using CAG within $48 \mathrm{~h}$ after admission, were selected for inclusion in this study. After obtaining informed consent, the 65 patients were randomly divided into those receiving drug treatment alone (drug group) and those receiving OCT-mediated treatment (OCT group). The drug group comprised 33 patients with an average age of $59.30 \pm 8.21$ years, including 23 men and 10 women. The OCT group consisted of 32 patients with an average age of $59.75 \pm 6.93$ years, including 26 men and 6 women. This 
study was conducted in accordance with the declaration of Helsinki. This study was conducted with approval from the Ethics Committee of Anhui Medical University. Written informed consent was obtained from all participants.

\section{Therapy for the Drug Group}

Under the premise of the absence of contraindications, the routine regimen of $100 \mathrm{mg} /$ day aspirin (Bayaspirin; Bayer HealthCare, Germany), $75 \mathrm{mg} /$ day clopidogrel (Plavix; Sanofi Winthrop Industries), statins, $\beta$-blockers, angiotensinconverting enzyme inhibitor (ACEI) or angiotensin receptor blocker (ARB), nitrates, and low molecular weight heparin (enoxaparin; Sanofi Winthrop Industries, France) were used during hospitalization for 8 days. In some patients, platelet glycoprotein IIb/IIIa receptor antagonist tirofiban (terbinafine; Lunan Bei Special Pharmaceutical Co, Shandong, China) was used.

\section{Therapy for the OCT Group}

OCT examination was performed immediately after CAG. Implantation of a sirolimus-eluting stent was performed if the OCT results suggested that the intermediate lesions were unstable plaques (plaque rupture, dissection, red blood clots, thin fibrous cap, or plaque neovascularization), whereas drug treatment was used for no promotion for unstable plaque. The drug treatment with drug-eluting stents implanted rapamycin or not was identical to the simple drug treatment group.

\section{Determination of the Extent of Coronary Artery Stenosis}

Quantitative CAG analysis (QCA) was performed by using an offline quantitative CAG system before and after stent implantation. By using a guiding catheter for magnification calibration, the diameters of the reference vessel (the average of the proximal and distal reference lumen diameters), the minimal luminal diameter, and the percentage diameter stenosis were measured before and after stenting from diastolic frames in a single, matched view showing the smallest minimal luminal diameter.

\section{OCT Operations and Analysis}

The radial artery approach was chosen for all patients, and the right radial artery was selected under the premise of the absence of contraindications according to the Allen trial. The left radial artery was selected if the puncture failed or if the right radial artery was abnormal. OCT examination was performed for the single vessel with stenosis (between $50 \%$ and $70 \%$ ), which is not part of the LMCA, by using CAG. Unfractionated heparin with a conventional dosage of $100 \mathrm{U} /$ $\mathrm{kg}$ was used. A 0.014 -in guidewire was inserted into a 3.2-F Helios blocking balloon catheter up to the distal end of the target vessels through a 6-F guiding catheter. The guidewire was withdrawn, and the imaging guidewire was sent to the target site and connected with the OCT system (US Lightlab Imaging). The Helios catheter was dilated to block the balloon in the proximal part of the target site with a low-pressure pump (0.3-1.0 atm), in order to block blood flow. Thereafter, Ringer solution was injected with a speed of $0.5-3.0 \mathrm{~mL} / \mathrm{s}$ into the coronary arteries by using the Helios blocking balloon
Table 1. Baseline Clinical Characteristics

\begin{tabular}{|c|c|c|c|}
\hline & Drug Group & OCT Group & $P$ \\
\hline Cases, $\mathrm{n}$ & 33 & 32 & \\
\hline Age, y & $59.30 \pm 8.21$ & $59.75 \pm 6.93$ & .81 \\
\hline Male, n (\%) & $23(69.70)$ & $26(81.25)$ & .28 \\
\hline Current smoking, n (\%) & $20(60.61)$ & $22(68.75)$ & .49 \\
\hline Hypertension, n (\%) & $18(54.55)$ & $20(62.50)$ & .52 \\
\hline Diabetes mellitus, n (\%) & $16(48.48)$ & $19(59.38)$ & .38 \\
\hline Prior myocardial infarction, $\mathrm{n}$ & 0 & 0 & \\
\hline Prior stroke, n (\%) & $3(9.09)$ & $2(6.25)$ & 1.00 \\
\hline Unstable angina, $\mathrm{n}(\%)$ & $18(54.55)$ & $14(43.75)$ & .38 \\
\hline Acute myocardial infarction, n (\%) & $15(45.45)$ & $18(56.25)$ & .38 \\
\hline TIMI risk score & $5.03 \pm 1.43$ & $4.69 \pm 1.49$ & .35 \\
\hline Middle-risk, n (\%) & $14(44.78)$ & $18(56.25)$ & .26 \\
\hline High-risk, n (\%) & $19(55.22)$ & $14(43.75)$ & .26 \\
\hline Hyperlipidemia, n (\%) & $15(45.45)$ & $12(37.50)$ & .51 \\
\hline Creatinine, $\mu \mathrm{mol} / \mathrm{L}$ & $82.94 \pm 19.56$ & $79.19 \pm 21.48$ & .46 \\
\hline Tnl, ng/mL & $4.67 \pm 6.31$ & $4.98 \pm 5.24$ & .83 \\
\hline Left ventricular ejection fraction, $\%$ & $51.85 \pm 10.45$ & $48.69 \pm 9.32$ & .20 \\
\hline Aspirin, n (\%) & $32(96.97)$ & $30(93.75)$ & .98 \\
\hline Clopidogrel, n (\%) & $33(100)$ & $32(100)$ & \\
\hline Statins, n (\%) & $30(90.91)$ & $31(96.88)$ & .63 \\
\hline$\beta$-blockers, n (\%) & $28(84.85)$ & $25(78.13)$ & .49 \\
\hline ACEI/ARB, n (\%) & $22(66.67)$ & $24(75.00)$ & .46 \\
\hline Low molecular weight heparin, $\mathrm{n}(\%)$ & $33(100)$ & $32(100)$ & \\
\hline Tirofiban, n (\%) & $8(24.24)$ & $15(46.88)$ & .06 \\
\hline
\end{tabular}

Values are expressed as mean \pm SD where indicated.

catheter. The OCT systems were started after clearing the target blood vessels. The imaging guidewire was automatically retracted with a speed of $3 \mathrm{~mm} / \mathrm{s}$, and dynamic imaging was performed, with a vascular length of $30-45 \mathrm{~mm}$ imaged at each time.

In accordance with the imaging standards, the plaques were divided into fibrous plaques, fiber calcified plaques, and fatty plaques. Fibrous plaques had a homogeneous strong signal area, fiber calcified plaques had a weak signal area with very clear boundary, and lipid-rich plaques showed a weak signal area with blurred edges [Yabushita 2002]. The fibrous cap was a strong signal zone covered by a weak signal area. The thickness of the thin fibrous cap was $\leq 65 \mu \mathrm{m}$. Plaque rupture was defined as the continuous breakage of the fibrous cap, forming a cavity in the plaque. White thrombus was defined as strong reflection signal organizations broke into the lumen, showing subsequently low attenuation and silent film. On the other hand, red thrombus showed rapid signal attenuation. Plaque neovascularization was defined as a lumen structure 
Table 2. Procedural Characteristics

\begin{tabular}{|c|c|c|c|}
\hline & Drug Group & OCT Group & $P$ \\
\hline \multicolumn{4}{|l|}{ Lesion location } \\
\hline LAD, n (\%) & $18(54.55)$ & $16(50.00)$ & .71 \\
\hline LCX, n (\%) & $8(24.24)$ & $5(15.63)$ & .39 \\
\hline RCA, n (\%) & $7(21.21)$ & $11(34.38)$ & .24 \\
\hline Type B2/C lesion, n (\%) & $11(33.33)$ & $14(43.75)$ & .39 \\
\hline Diameter stenosis (\%) & $60.70 \pm 2.58$ & $61.91 \pm 3.18$ & .10 \\
\hline Reference vessel diameter $(\mathrm{mm})$ & $3.37 \pm 0.43$ & $3.40 \pm 0.43$ & .79 \\
\hline \multicolumn{4}{|l|}{ Minimal lumen diameter $(\mathrm{mm})$} \\
\hline Preintervention & $2.05 \pm 0.28$ & $2.09 \pm 0.30$ & .50 \\
\hline Postintervention & & $3.03 \pm 0.36$ & \\
\hline Number of stents & & $1.29 \pm 0.46$ & \\
\hline Stent diameter $(\mathrm{mm})$ & & $3.10 \pm 0.40$ & \\
\hline Total stent length $(\mathrm{mm})$ & & $32.96 \pm 14.83$ & \\
\hline \multicolumn{4}{|l|}{ Tnl in OCT no PCl, ng/mL } \\
\hline \multicolumn{4}{|l|}{ Pre-OCT } \\
\hline $2.86 \pm 6.04$ & .96 & & \\
\hline Post-OCT & & $2.73 \pm 5.67$ & \\
\hline
\end{tabular}

Values are expressed as mean $\pm S D$ where indicated. $L A D$ indicates left anterior descending artery; LCX, circumflex artery; RCA, right coronary artery; OCT, optical coherence tomography.

with no communication with the lumen and no signal (three or more frames) [Di Stefano 2009; Russell 2008].

\section{Observation Indicators}

The following were observed and recorded: the baseline clinical and procedural characteristics, the average length of stay, hospital stay, and MACEs (including death, myocardial infarction, and stroke) in the follow-up period (1, 3, 6, and 12 months after January by telephone or outpatient followup mode) of the patients in the two groups; the revascularization received in the drug group during follow-up; OCT examination success rate and related complications; implantation of drug-eluting stents; and repeated target vessel revascularization.

\section{Statistical Analysis}

Categorical variables were compared with the Pearson chi-square test or Fisher exact test, and continuous variables, expressed as mean $\pm \mathrm{SD}$, were compared with Student $\mathrm{t}$ test. Kaplan-Meier estimates were used to present cumulative incidence data with between-group comparisons by using the log-rank test. Cox proportional hazard regression was used to estimate hazard ratios and their $95 \%$ confidence intervals. All statistical analyses were completed with SAS software (version 8.2 or higher; SAS Institute, Cary, NC, USA). $P<.05$ was considered statistically significant.

\section{RESULTS}

The indicators of age, sex ratio, smoking, hypertension, diabetes, prior myocardial infarction, prior stroke, TIMI risk score, hyperlipidemia, creatinine, TnI, left ventricular ejection fraction, use of drugs (aspirin, clopidogrel, statins, $\beta$-blockers, ACEI or ARB class, low molecular weight heparin, nitrates, and tirofiban), and the angiographic and procedural characteristics (lesion location, lesion type, diameter stenosis, reference vessel diameter, and minimal lumen diameter) showed no statistically significant differences (Tables 1 and 2).

\section{Average Length of Stay, Revascularization, and MACEs}

There were no significant differences in the average length of stay of patients. No deaths or stroke occurred during hospitalization and follow-up. However, during follow-up, 11 patients ( 6 due to frequent angina and 5 due to acute myocardial infarction) in the drug group were hospitalized again and underwent CAG and PCI as well as sirolimus-eluting stent implantation. Although two patients in the OCT group had repeat revascularization (new lesion), no myocardial infarction occurred. Both showed a significant difference $(P<.05)$ (Table 3, Figures 1 and 2$)$.

\section{OCT Examination and Related Conditions}

All 32 patients in the OCT group successfully completed the inspection work related to vessels. Twelve patients had varying degrees of chest tightness in the process of using the Helios blocking balloon to occlude blood flow. The electrocardiogram (ECG) showed a transient decrease in heart rate and blood pressure (often occurring in the right coronary artery or circumflex artery dissection), which improved without special treatment soon after the lifting of blocking. One older woman (72 years old) was implanted with an additional stent in the process of using the Helios blocking balloon to block the proximal part of the right coronary artery. The OCT examination results of 24 of 32 patients indicated unstable plaques, for which implantations of sirolimus-eluting stents were performed; of these patients, 18 had plaque rupture or dissection, 4 had red thrombus, 5 had a thin fibrous cap, and 5 had plaque neovascularization. Although OCT plaques were found in eight patients, the fibrous cap thickness was $>80 \mu \mathrm{m}$, and no signs of plaque rupture and thrombosis appeared; thus, they did not receive further intervention.

\section{DISCUSSION}

ACS is a clinical emergency caused by varying degrees of luminal obstruction resulting from the formation of secondary thrombosis based on vulnerable plaques in coronary heart disease, including UA, NSTEMI, STEMI, and sudden death. Policy guidelines recommended the treatment of NSTEACS (UA/NSTEMI) patients based on risk stratification. In recent years, evidence-based medicine [Jneid 2012] has shown that early intervention therapy for UA/NSTEMI patients of middle and high risk can significantly reduce the occurrence of adverse cardiovascular events (ACEs) such as death, myocardial infarction, and stroke. Most 


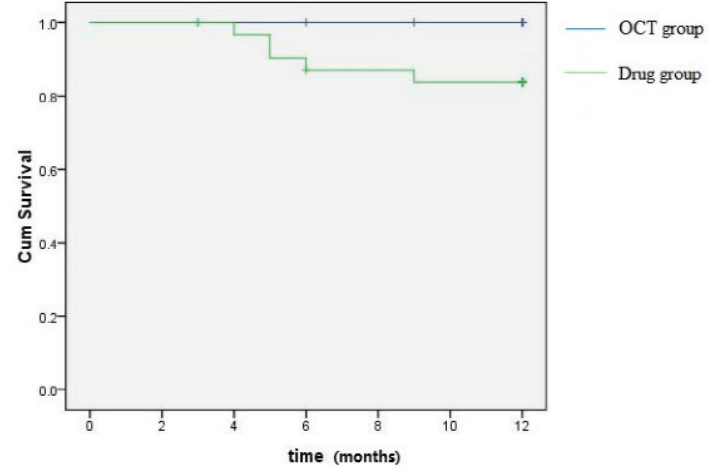

Figure 1. Kaplan-Meier 1-year MI-free survival.

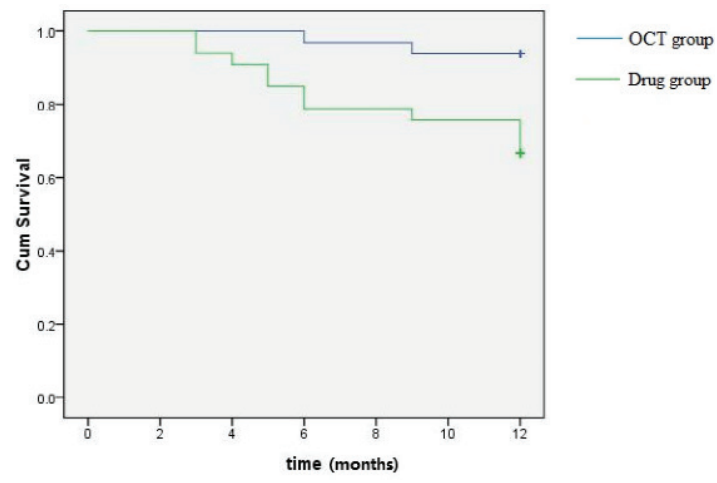

Figure 2. Kaplan-Meier 1-year revascularization-free survival.

scholars considered a vascular stenosis of $\geq 75 \%$ on $C A G$ as an indication for PCI. It has been controversial as to whether or not interventional therapy should be used for UA/NSTEMI patients in the middle- and high-risk groups, with vessel diameter stenosis at the critical level (50\%-70\%). Conventionally, intervention is not recommended and conservative medical treatment is preferred. However, in recent years, studies have shown findings of a high incidence of ACEs in patients receiving conservative drug treatment in long-term followups [Rzeczuch 2005]. In this study, during the follow-up, the patients in the drug group were rehospitalized and underwent revascularization because myocardial infarction and angina increased significantly, which also supported the poor efficacy of drug treatment alone. For these patients, although the degree of coronary artery stenosis was not high, vulnerable plaques may be present. Vulnerable plaques were not effectively identified by using the clinical results and QCA. Therefore, how to establish early diagnosis and perform early treatment for these patients with intermediate lesions-who are likely to be at a higher risk in the future-has become an important issue in modern medicine.

OCT tomography is performed to investigate the internal structure of living tissues by using the short coherence properties of a broadband light source. The basic principle is similar to that of conventional ultrasound imaging. Different from IVUS, the reflected infrared light in OCT is captured through the low-coherence near-infrared beam perpendicular
Table 3. Analysis of Hospital Days and 1-Year Clinical Outcomes

\begin{tabular}{lccccc}
\hline Droup & $\begin{array}{c}\text { Revascu- } \\
\text { Hospital }(\mathrm{n})\end{array}$ & $\begin{array}{c}\text { larization } \\
\text { (cases) }\end{array}$ & $\begin{array}{c}\text { Stroke } \\
\text { (cases) }\end{array}$ & $\begin{array}{c}\text { Myocardial } \\
\text { Infarction } \\
\text { (cases) }\end{array}$ & $\begin{array}{c}\text { Deaths } \\
\text { (cases) }\end{array}$ \\
\hline $\begin{array}{l}\text { Drug } \\
\text { group } \\
\text { OCT } \\
\text { group }\end{array}$ & $8.88 \pm 0.99$ & $11 *$ & 0 & $5^{*}$ & 0 \\
\hline
\end{tabular}

Compared with the OCT group, ${ }^{*}<<.05$. Myocardial infarction: $\chi^{2}=5.533$, $P<.05$; Revascularization: $\chi^{2}=7.445, P<.01$.

to the axis of the catheter emitted by an ultra-light-emitting diode; then, the depth is measured by using the interference method for image reconstruction [Huang 1991] with a high resolution (10 times higher than the IVUS resolution). The axial resolution is $10-15 \mu \mathrm{m}$, whereas the vertical resolution is $20-25 \mu \mathrm{m}$. OCT could clearly show atherosclerotic plaques from the organizational level, thereby providing information about small coronary structure changes without sample removal or treatment. Therefore, OCT is also known as "histological microscopy of the body," [Honda 2008] which is a recent medical diagnostic ultrasound imaging technology after radiology, CT, and MRI. Jang et al, at Harvard University, applied OCT for intracoronary examination for the first time in 2000 [Jang 2002]; thereafter, the method has received rapidly growing popularity worldwide owing to its security and high resolution.

Naghavi et al [Naghavi 2003] reported that the main criteria for vulnerable plaques are acute plaque inflammation, a large lipid core, a thin fibrous cap, endothelial loss with platelet aggregation on the surface, plaque rupture, and stenosis $>90 \%$ according to an autopsy study. The basic lesion of plaque rupture is described morphologically as a thin fibrous cap of the plaque (TCFA). With its high resolution, OCT can accurately measure the thickness of the fibrous cap [Kume 2006a], as well as identify plaques [Yabushita 2002] and thrombosis. [Kume 2006b]. Generally, TCFA on OCT evaluation is defined as a plaque with two quadrants of lipid area greater than or equal to the vascular circumference, with a fibrous cap thickness of $<65 \mu \mathrm{m}$. Jang et al [Jang 2005] found that vulnerable plaques with a fibrous cap thickness of $\leq 65 \mu \mathrm{m}$ accounted for $72 \%$ in acute myocardial infarction patients, $50 \%$ in ACS patients, and only $20 \%$ in patients with stable angina pectoris. More autopsy cases proved that the fibrous cap thickness of $95 \%$ of ruptured plaques was $<65 \mu \mathrm{m}$. In recent years, studies have shown [Kitabata 2010] that plaque neovascularization is another sign of plaque vulnerability, which can indirectly promote plaque instability by transporting inflammatory cells, even promoting rupture.

In this study, 32 UA/NSTEMI patients with intermediate lesions in the middle- and high-risk groups were immediately subjected to OCT examination after CAG, similar to the above studies. It was found that 24 patients had vulnerable 
plaques, 18 had plaque rupture or dissection, 4 had red thrombus, 5 had thin fibrous cap, and 5 had plaque neovascularization. These patients were treated with PCI and implanted with rapamycin-eluting stents. On the other hand, the other eight patients without vulnerable plaques on OCT were given only simple drug therapy. Furthermore, no MACE occurred during hospitalization and the 1-year follow-up period in the patients of this group, which showed statistical significance with respect to the 33 patients of the drug group. This suggests that the risk of drug therapy alone for MACEs in UA/ NSTEMI patients with intermediate lesions of the middle- or high-risk group was larger; however, choosing the treatment strategy under the premise of further assessment for vulnerable plaques by using OCT may be more scientifically sound.

In terms of safety, all OCT examinations were successfully completed. Twelve patients felt varying degrees of chest tightness in the process of using the Helios blocking balloon to block the blood flow. The ECG of some patients showed a decrease in heart rate and blood pressure (occurring in the right coronary artery or circumflex artery), considering the relationship with myocardial ischemia when Ringer solution was used to clean the vessels in the process of briefly blocking the blood flow with the balloon. However, the situation can quickly improve with no special treatment. In the early use of OCT, one older female patient (72 years old) had dissection and received additional stent implantation in the process of using the Helios blocking balloon for proximal right coronary occlusion. This result may be related to the patient's own blood vessel conditions; however, another possibility may be the insufficient learning curve in the early stages of OCT operation. The pump intensity was too large to exceed the size of the balloon, filling the patient's vessel diameter when applying pressure to block the Helios balloon. No similar complications occurred in the later operation by strictly controlling the expansion pressure of the pressure pump.

The main limitation of this study was that the firstgeneration OCT (time domain OCT) had many technical deficiencies. The specific limitations in clinical applications were as follows [Honda 2008]: (i) atherosclerotic plaques judged by using OCT were influenced by their poor penetration, and full vessel pictures could not be obtained for coronary arteries $>3.5 \mathrm{~mm}$ in diameter; (ii) the blood can cause signal attenuation and scatter; therefore, a low-pressure balloon was needed in OCT to block the forward blood flow, whereas contrast agents were used to scour the blood vessels and relatively shorten the imaging time; (iii) the temporary blocking of the forward blood flow was the most important drawback in the evaluations, as it could result in myocardial ischemia, and blocking the balloon would damage the vascular endothelium, resulting in vascular dissection-this increases the risk of the operation, which is contraindicated for patients with left main lesions, opening lesions, and heart dysfunction. The scanning speed and time were significantly reduced for the new generation of frequency-domain OCT. Images can be successfully obtained without blocking blood flow but by only using a bolus injection of a contrast agent $(3-4 \mathrm{~mL} / \mathrm{s})$, significantly reducing the adverse reactions [Gonzalo 2010]. Left main lesions, opening lesions, and vessels with a diameter of $>3.5 \mathrm{~mm}$ are eligible for vascular imaging [Bezerra 2009]. This study shows that the use of OCT to guide therapeutic strategies in ACS patients with intermediate lesions may have broader applications.

\section{REFERENCES}

Abizaid A, Mintz GS, Pichard AD, et al. 1998. Clinical intravascular ultrasound and quantitative angiographic determinants of the coronary flow reserve before and after percutaneous transluminal coronary angioplasty. Am J Cardiol 82:423-8.

Bech GJ, De Bruyne B, Pijls NH, et al. 2001. Fractional flow reserve to determine the appropriateness of angioplasty in moderate coronary stenosis: a randomized trial. Circulation 103:2928-34.

Bezerra HG, Costa MA, Guagliumi G, Rollins AM, Simon DI. 2009. Intracoronary optical coherence tomography: a comprehensive review clinical and research applications. JACC Cardiovasc Interv 2:1035-46.

Briguori C, Anzuini A, Airoldi F, et al. 2001. Intravascular ultrasound criteria for the assessment of the functional significance of intermediate coronary artery stenosis and comparison with fractional flow reserve. Am J Cardiol 87:136-41.

De Bruyne B, Pijls NH, Kalesan B, et al. 2012. Fractional flow reserve guided PCI versus medical therapy in stable coronary disease. N Engl J Med 367:991-1001.

Di Stefano R, Felice F, Balbarini A. 2009. Angiogenesis as risk factor for plaque vulnerability. Curr Pharm Des 15:1095-1106.

Fernandes MR, Silva GV, Caixeta A, Rati M, de Sousa e Silva NA, Perin EC. 2007. Assessing intermediate coronary lesions: angiographic prediction of lesion severity on intravascular ultrasound. J Invasive Cardiol 19:412-16

Glaser R, Selzer F, Faxon DP, et al. 2005. Clinical progression of incidental, asymptomatic lesions discovered during culprit vessel coronary intervention. Circulation 111:143-9.

Goldman L, Schafer AI. 2012. Goldman's Cecil Medicine (24th Edition). Elsevier (Singapore) Pte Ltd. 1:425-53.

Gonzalo N, Tearney GJ, Serruys PW, et al. 2010. Second-generation optical coherence tomography in clinical practice. High-speed data acquisition is highly reproducible in patients undergoing percutaneous coronary intervention. Rev Esp Cardiol 63:893-903.

Honda Y, Fitzgerald PJ. 2008. Frontiers in intravascular imaging technologies. Circulation 117:2024-37.

Huang D, Swanson EA, Lin CP, et al. 1991. Optical coherence tomography. Science 254:1178-81.

Jang IK, Bouma BE, Kang DH, et al. 2002. Visualization of coronary atherosclerotic plaques in patients using optical coherence tomography: comparison with intravascular ultrasound. J Am Coll Cardiol 39:604-9.

Jang IK, Tearney GJ, MacNeill B, et al. 2005. In vivo characterization of coronary atherosclerotic plaque by use of optical coherence tomography. Circulation 111:1551-5.

Jneid H, Anderson JL, Wright RS, et al. 2012. 2012 ACCF/AHA focused update of the guideline for the management of patients with unstable angina/Non-ST-elevation myocardial infarction (updating the 2007 guideline and replacing the 2011 focused update): a report of the American College of Cardiology Foundation/American Heart Association Task Force on practice guidelines. Circulation 126:875-910.

Kitabata H, Tanaka A, Kubo T, et al. 2010. Relation of microchannel 
structure identified by optical coherence tomography to plaque vulnerability in patients with coronary artery disease. Am J Cardiol 105:1673-8.

Kume T, Akasaka T, Kawamoto T, et al. 2006. Assessment of coronary arterial plaque by optical coherence tomography. Am J Cardiol. 97:1172-5.

Kume T, Akasaka T, Kawamoto T, et al. 2006. Assessment of coronary arterial thrombus by optical coherence tomography. Am J Cardiol 97:1713-17.

Naghavi M, Libby P, Falk E, et al. 2003. From vulnerable plaque to vulnerable patient: a call for new definitions, risk assessment strategies: Part II. Circulation 108:1772-8.

Russell DA, Abbott CR, Gough MJ. 2008. Vascular endothelial growth factor is associated with histological instability of carotid plaques. Br J Surg 95:576-81.
Rzeczuch K, Szajn G, Jankowska E, et al. 2005. Borderline coronary lesions may lead to serious coronary events-long-term outcome in 65 conservatively treated patient. Kardiol Pol 63:50-7.

Takagi A, Tsurumi Y, Ishii Y, Suzuki K, Kawana M, Kasanuki H. 1999. Clinical potential of intravascular ultrasound for physiological assessment of coronary stenosis: relationship between quantitative ultrasound tomography and pressure-derived fractional flow reserve. Circulation $100: 250-5$

Tonino PA, De Bruyne B, Pijls NH, et al. 2009. Fractional flow reserve versus angiography for guiding percutaneous coronary intervention. $\mathrm{N}$ Engl J Med 360:213-24.

Yabushita H, Bouma BE, Houser SL, et al. 2002. Characterization of human atherosclerosis by optical coherence tomography. Circulation 106:1640-5. 